\title{
Separating cosmic shear from intrinsic galaxy alignments: Correlation function tomography
}

\author{
L. J. King and P. Schneider \\ Institut für Astrophysik und Extraterrestrische Forschung, Universität Bonn, Auf dem Hügel 71, 53121 Bonn, Germany \\ Received 23 September 2002 / Accepted 22 October 2002

\begin{abstract}
During the past few years, secure detections of cosmic shear have been obtained, manifest in the correlation of the observed ellipticities of galaxies. Constraints have already been placed on cosmological parameters, such as the normalisation of the matter power spectrum $\sigma_{8}$. One possible systematic contaminant of the lensing correlation signal arises from intrinsic galaxy alignment, which is still poorly constrained. Unlike lensing, intrinsic correlations only pertain to galaxies with small physical separations, the correlation length being a few Mpc. We present a new method that harnesses this property, and isolates the lensing and intrinsic components of the galaxy ellipticity correlation function using measurements between different redshift slices. The observed signal is approximated by a set of template functions, making no strong assumptions about the amplitude or correlation length of any intrinsic alignment. We also show that the near-degeneracy between the matter density parameter $\Omega_{\mathrm{m}}$ and $\sigma_{8}$ can be lifted using correlation function tomography, even in the presence of an intrinsic alignment signal.
\end{abstract}

Key words. cosmology: dark matter - cosmology: observations - methods: data analysis

\section{Introduction}

The tidal gravitational field of mass inhomogeneities distorts the images of distant galaxies, resulting in correlations in their observed ellipticities. This cosmological weak lensing signal, or cosmic shear, depends upon cosmological parameters and the matter power spectrum (Blandford et al. 1991; Miralda-Escudé 1991; Kaiser 1992). In 2000, four teams announced the first detections of cosmic shear (Bacon et al. 2000; Kaiser et al. 2000; van Waerbeke et al. 2000; Wittman et al. 2000; Maoli et al. 2001), and more recently measurements at arcminute scales have been made using the HST (Hämmerle et al. 2002; Refregier et al. 2002). Interesting constraints have already been placed on the matter power spectrum normalisation $\sigma_{8}$, and cosmic shear is also particularly sensitive to the matter density parameter $\Omega_{\mathrm{m}}$, the power spectrum shape parameter $\Gamma$ and the source redshift distribution (e.g. van Waerbeke et al. 2002a; Hoekstra et al. 2002). Future multi-colour surveys will cover hundreds of square degrees, and have the potential to place tight constraints on cosmological parameters particularly when combined with results from the CMB, SNIa and galaxy surveys (Mellier et al. 2002; van Waerbeke et al. 2002b). For example, van Waerbeke et al. (2002a) compared their constraints on $\Omega_{\mathrm{m}}$ and $\sigma_{8}$ from lensing with those of Lahav et al. (2002) from the CMB, noting their near orthogonality.

Various statistical measures of the cosmic shear have been suggested; here we focus on the two-point shear correlation

Send offprint requests to: L. J. King, e-mail: lindsay@astro.uni-bonn.de function $\xi_{+}$(hereafter denoted by $\xi$ ), which is convenient since it is insensitive to gaps in the data field, unlike integrated measures such as the aperture mass statistic $\mathcal{M}_{\text {ap }}$ (e.g. Schneider et al. 1998).

A possible systematic contaminant of the lensing correlation function $\xi^{\mathrm{L}}$ is intrinsic alignment, which may arise during the galaxy formation process. This has been subject to numerical, analytic and observational studies (e.g. Croft \& Metzler 2000; Heavens et al. 2000 (HRH); Crittenden et al. 2001; Catelan et al. 2001; Mackey et al. 2002; Brown et al. 2002; Jing 2002; Hui \& Zhang 2002), where amplitude estimates span a few orders of magnitude due to differences in the mechanism assumed to be responsible, and the type of galaxy considered. Nevertheless, these studies agree that the intrinsic correlation signal $\xi^{\mathrm{I}}$ can dominate the lensing signal for surveys with $\langle z\rangle \lesssim 0.5$.

Any correlation in ellipticities due to intrinsic alignment only arises from physically close galaxy pairs, whereas $\xi^{\mathrm{L}}$ is sensitive to the integrated effect of the density fluctuations out to the redshift of the nearer galaxy. It has been shown that photometric redshift information could be used to suppress $\xi^{\mathrm{I}}$, by downweighting or ignoring galaxy pairs at approximately the same redshift (King \& Schneider 2002), or by downweighting nearby pairs and subtracting a model of the intrinsic alignment signal from the observed ellipticity correlation function (Heymans \& Heavens 2002).

Motivated by the fact that intrinsic galaxy alignment is not yet well understood, we present a new method to isolate the intrinsic and lensing-induced components of the galaxy 
ellipticity correlation function. This method assumes that photometric redshift information is available, so that the correlation function can be measured between different redshift slices. However, no specific model for intrinsic ellipticity correlation (for instance its correlation length or redshift evolution) needs to be adopted. In the next section we outline the method and in Sect. 3 we present some results in the context of a possible future survey. We discuss the results in Sect. 4.

\section{General method and specific assumptions}

In this section we outline a method to separate and extract the intrinsic and lensing components of the galaxy ellipticity correlation function. The general method is described in Sect. 2.1, and in Sects. 2.2 and 2.3 we state the assumptions particular to this work.

\subsection{Method}

The ellipticity correlation function for galaxies with angular separation $\theta$ and at true redshifts $z_{i}, z_{j}$, is composed of a lensing and an intrinsic signal

$\xi\left(\theta, z_{i}, z_{j}\right)=\xi^{\mathrm{L}}\left(\theta, z_{i}, z_{j}\right)+\xi^{\mathrm{I}}\left(\theta, z_{i}, z_{j}\right)$.

As noted in Sect. 1, the origin and behaviour of $\xi^{\mathrm{I}}$ is not yet well understood. $\xi^{\mathrm{L}}\left(\theta, z_{i}, z_{j}\right)$ is related to the 3-dimensional matter power spectrum $P_{\delta}$ through

$$
\begin{aligned}
& \xi^{\mathrm{L}}\left(\theta, z_{i}, z_{j}\right)=\frac{9 H_{0}^{4} \Omega_{\mathrm{m}}^{2}}{4 c^{4}} \int_{0}^{\min \left[w_{i}, w_{j}\right]} \frac{\mathrm{d} w}{a^{2}(w)} \\
& \quad \times R\left(w, w_{i}\right) R\left(w, w_{j}\right) \int \frac{\mathrm{d} \ell \ell}{(2 \pi)} P_{\delta}\left(\frac{\ell}{f(w)}, w\right) J_{0}(\ell \theta),
\end{aligned}
$$

where $H_{0}$ and $\Omega_{\mathrm{m}}$ are the values of the Hubble parameter and matter density parameter at the present epoch, and $a(w)$ is the scale factor at comoving distance $w$, normalised such that $a(0)=1$ today. $J_{0}$ is the 0 th order Bessel function of the first kind, and $\boldsymbol{\ell}$ is the angular wave-vector. We denote the comoving distance at $z_{i}$ by $w_{i}$, and the function $f(w)$ is the comoving angular diameter distance, which depends on the spatial curvature $K$ :

$$
f(w)=\left\{\begin{array}{ll}
K^{-1 / 2} \sin \left(K^{1 / 2} w\right) & (K>0) \\
w & (K=0) \\
(-K)^{-1 / 2} \sinh \left[(-K)^{1 / 2} w\right] & (K<0)
\end{array}\right\} .
$$

The function $R\left(w, w^{\prime}\right)=f\left(w^{\prime}-w\right) / f\left(w^{\prime}\right)$ is the ratio of the angular diameter distance of a source at comoving distance $w^{\prime}$ seen from a distance $w$, to that seen from $w=0$.

Next, we account for the availability of photometric redshift estimates rather than spectroscopic ones. The galaxy ellipticity correlation function becomes

$\bar{\xi}\left(\theta, \bar{z}_{i}, \bar{z}_{j}\right)=\int \mathrm{d} z_{i} \int \mathrm{d} z_{j} p\left(z_{i}, z_{j} \mid \bar{z}_{i}, \bar{z}_{j}, \theta\right) \xi\left(\theta, z_{i}, z_{j}\right)$,

where $p\left(z_{i}, z_{j} \mid \bar{z}_{i}, \bar{z}_{j}, \theta\right)$ is the probability to have true redshifts $z_{i}$ and $z_{j}$ given photometric estimates $\bar{z}_{i}$ and $\bar{z}_{j}$. This is given by

$$
p\left(z_{i}, z_{j} \mid \bar{z}_{i}, \bar{z}_{j}, \theta\right)=\frac{p\left(z_{i} \mid \bar{z}_{i}\right) p\left(z_{j} \mid \bar{z}_{j}\right)\left[1+\xi_{\mathrm{gg}}(r)\right]}{\int \mathrm{d} z_{i} \int \mathrm{d} z_{j} p\left(z_{i} \mid \bar{z}_{i}\right) p\left(z_{j} \mid \bar{z}_{j}\right)\left[1+\xi_{\mathrm{gg}}(r)\right]},
$$

where $\xi_{\text {gg }}$ is the galaxy spatial correlation function, which may also include redshift dependent evolution, and $r$ is the comoving separation of the galaxies in a pair.

We now assume that $\bar{\xi}\left(\theta, \bar{z}_{i}, \bar{z}_{j}\right)$ is available on a 3-dimensional grid of $N_{K}$ angular separation bins of width $\Delta \theta$ centred on $\theta_{K}$ (index $K$ ), and $N_{Z}$ photometric redshift bins of width $\Delta z$ centred on each of $\bar{z}_{i}$ (index $I$ ) and $\bar{z}_{j}$ (index $J$ ). This could either correspond to an observed signal $\bar{\xi}_{I J K}^{\text {obs }}$, or to a theoretical prediction $\left\langle\bar{\xi}^{\text {mod }}\right\rangle_{I J K}$ which we want to compare with the observed signal.

We assume that both the lensing and intrinsic correlations can be written in terms of sets of template functions $A_{n}$ and $B_{n}$

$\begin{aligned} \bar{\xi}^{\mathrm{L}}\left(\theta, \bar{z}_{i}, \bar{z}_{j}\right) & =\sum_{n=1}^{\mathrm{N}_{\mathrm{L}}} a_{n} A_{n}\left(\theta, \bar{z}_{i}, \bar{z}_{j}\right), \\ \bar{\xi}^{\mathrm{I}}\left(\theta, \bar{z}_{i}, \bar{z}_{j}\right) & =\sum_{n=1}^{\mathrm{N}_{\mathrm{I}}} b_{n} B_{n}\left(\theta, \bar{z}_{i}, \bar{z}_{j}\right),\end{aligned}$

where $a_{n}$ and $b_{n}$ are the amplitudes of the $n$th lensing and $n$th intrinsic template functions. The template functions are fairly arbitrary, and extra functions can be added as required, to span the range of plausible models. We describe our choice of models in Sect. 2.3 below.

A suitably chosen single index $m$ identifies correlations between bins with redshift indices $I, J$ and angular separation index $K$. In total, there are $N_{M}=N_{Z}\left(N_{Z}+1\right) N_{K} / 2$ such independent measurements. The total of $N=N_{L}+N_{I}$ gridded template models for the correlation functions $\left(A_{1} \ldots A_{N_{L}}, B_{1} \ldots B_{N_{I}}\right)$ can be written as an $N_{M} \times N$ so-called design matrix $\mathcal{M}$, and their amplitudes $\left(a_{1} \ldots a_{N_{L}}, b_{1} \ldots b_{N_{I}}\right)$ as an $N$-dimensional column vector $\mathcal{G}$ so that

$\left\langle\bar{\xi}^{\mathrm{mod}}\right\rangle_{m}=\mathcal{M}_{m n} \mathcal{G}_{n}$

Our aim is to recover $\bar{\xi}_{m}^{\text {obs }}$ in terms of the template functions. Using the method of least squares, the best-fit estimates of the $\mathcal{G}_{n}$ are those values $\hat{\mathcal{G}}_{n}$ which minimise

$S=\left(\bar{\xi}^{\text {obs }}-\mathcal{M G}\right) C^{-1}\left(\bar{\xi}^{\mathrm{obs}}-\mathcal{M G}\right)$,

where $C$ is the covariance matrix. Since $\left\langle\bar{\xi}^{\text {mod }}\right\rangle_{m}$ is a linear combination of template functions, the linear least squares estimators are

$\hat{\mathcal{G}}=\left(\mathcal{M}^{T} C^{-1} \mathcal{M}\right)^{-1} \mathcal{M}^{T} C^{-1} \overline{\boldsymbol{\xi}}^{\mathrm{obs}}$.

After obtaining $C$, combined with an observed correlation function $\overline{\boldsymbol{\xi}}^{\text {obs }}$ and a design matrix $\mathcal{M}$, we have the necessary machinery to obtain $\hat{\mathcal{G}}$ i.e. the projection of $\overline{\boldsymbol{\xi}}^{\text {obs }}$ into the template functions. This means that separate fits for the lensing and intrinsic contributions can be obtained.

\subsection{Covariance matrix}

To evaluate (9), we need the covariance matrix $C_{m m^{\prime}}$. The covariance matrix could be calculated using the method described in Schneider et al. (2002), where $C$ is expressed as integrals over (products of) correlation functions. However, since the method presented here would require the calculation of very 


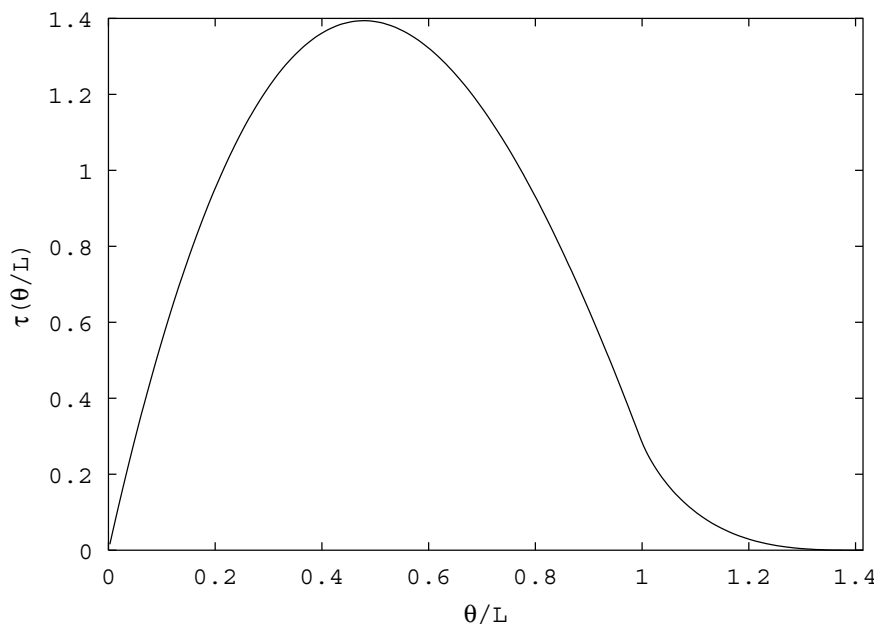

Fig. 1. The function $\tau(\theta / L)$ (as defined in the text) which accounts for the finite extent of the data field when calculating the number of pairs in a given bin.

many elements of the covariance matrix, owing to the redshift slicing, we decided to use, as a first step, a simplified model for $C$. This consists of neglecting the cosmic variance contribution to $C$, and thus consideration of the (diagonal) elements of $C$ coming from the intrinsic ellipticity dispersion of the source galaxies. This is the dominant contribution to the covariance at small angular scales; at larger angular scales, the cosmic variance terms start to dominate, with the transition angular scale depending on the survey geometry (Kaiser 1998; Schneider et al. 2002). Here, we consider $\mathcal{N}_{\mathcal{F}}$ independent fields, and take $\mathcal{N}_{\mathcal{F}}=300$. Therefore, we expect the cosmic variance not to be very much larger than the intrinsic ellipticity noise on the angular scales considered.

With this approximation, the elements of the covariance matrix are

$C_{m m^{\prime}}=\frac{2\left(\sigma_{\epsilon}^{2} / 2\right)^{2}}{\mathcal{N}_{\mathrm{p}}(m)}\left[\delta_{m, m^{\prime}}\left(1+\delta_{I, I^{\prime}} \delta_{J, J^{\prime}} \delta_{I, J}\right)\right]$,

where there is an extra contribution from auto-variance terms. The elements of the inverse covariance matrix in this case are simply $\left(C^{-1}\right)_{m m^{\prime}}=\delta_{m m^{\prime}} / C_{m m}$. The galaxy ellipticity dispersion is denoted by $\sigma_{\epsilon}$. In bin $m$, the number of pairs is given by

$\mathcal{N}_{\mathrm{p}}(m)=\mathcal{N}_{\mathrm{F}}\left[n_{0} p\left(z_{I}\right) \Delta z\right]\left[n_{0} p\left(z_{J}\right) \Delta z\right] L^{4} \frac{\Delta \theta}{L} \tau\left(\frac{\theta}{L}\right)$,

where $n_{0}$ is the galaxy number density, $p\left(z_{I}\right)$ is the redshift probability density for redshift bin $I$, and $L$ is the extent of the field, assumed to be square. $\tau(\theta / L)$ is a function that takes into account the fact that fields have finite extent, and it must be evaluated numerically. Figure 1 shows the function $\tau(\theta / L)$; note the limiting case where pairs separated by more than $\sqrt{2} L$ do not occur.

\subsection{Basis models}

In order to illustrate the general method described above, we will choose a simple, restricted set of template functions which share the approximate functional behaviour expected from the real correlation functions, both intrinsic and lensing. For the latter, we simply take a small number of CDM cosmologies and consider their correlation functions as a template set. For the former, simple exponentials with redshift dependence are chosen.

The lensing template functions $A_{n}$ used here are the gridded $\bar{\xi}^{\mathrm{L}}\left(\theta, \bar{z}_{i}, \bar{z}_{j}\right)$ for 3 models of the underlying cosmology: $\Lambda \mathrm{CDM}$ $\left(\Omega_{\mathrm{m}}=0.3, \Omega_{\Lambda}=0.7\right), \operatorname{OCDM}\left(\Omega_{\mathrm{m}}=0.3, \Omega_{\Lambda}=0\right)$ and $\sigma \mathrm{CDM}$ $\left(\Omega_{\mathrm{m}}=1, \Omega_{\Lambda}=0\right)$; for simplicity, $\sigma_{8}=0.9$ and $\Gamma=0.21$ for each model. We use the Bardeen et al. (1986) transfer function to describe the evolution of the 3-dimensional power spectrum, along with the prescription of Peacock \& Dodds (1996) for evolution in the non-linear regime. The required lensing correlation functions are calculated using the relationship between the power spectrum and $\xi^{\mathrm{L}}$ given in (2), and then integrated over the photometric redshift uncertainties as in (4). Here it is assumed that $p(z \mid \bar{z})$ is a Gaussian with dispersion $\sigma_{\text {phot }}$, centred on $\bar{z}$.

Nine template models $B_{n}$ for the intrinsic alignments are considered. First, the true spatial intrinsic correlation function is parameterised in terms of a correlation length $R_{\text {corr }}$ and an exponent $\alpha$ :

$\eta(r, z)=\left(1+z_{\mathrm{av}}\right)^{\alpha}\left[\exp \left(-r / R_{\mathrm{corr}}\right)\right]$

where $z_{\mathrm{av}}$ is the mean redshift of galaxies in a pair and $r$ is their comoving separation. We use the approximation $r^{2}=$ $\left(w_{i}-w_{j}\right)^{2}+\theta^{2} f^{2}\left[\left(w_{i}+w_{j}\right) / 2\right] . R_{\text {corr }}$ was taken to be $[1,3$, $10] h^{-1} \mathrm{Mpc}$ and $\alpha$ to be $[-1,0,1]$. The availability of photometric redshift estimates is then accounted for by integrating $\eta(r, z)$ as in (4), and finally we obtain each of the model correlation functions on a grid. Note that the intrinsic models are calculated using the relationships for the distances $f(w)$ pertaining to the $\Lambda \mathrm{CDM}$ cosmology described above, and that we use $\xi_{\mathrm{gg}}(r)=\left(r / 5 h^{-1} \mathrm{Mpc}\right)^{-1.8}$.

\section{Results}

The results are presented in the context of a possible future multi-colour cosmic shear survey, with a field size $L=14^{\prime}$, and $\mathcal{N}_{\mathcal{F}}=300$ independent pointings (i.e. the largest scale on which the ellipticity correlation function is available is $\sqrt{2} \times 14^{\prime}$ ). A galaxy number density of $30 \mathrm{arcmin}^{-2}$ and ellipticity dispersion of $\sigma_{\epsilon}=0.3$ are used throughout. The value of $\sigma_{\text {phot }}=0.1$ is chosen, typical of that obtained with current SED fitting procedures such as hyper- $Z$ using a wide range of optical and near-infrared filters (Bolzonella et al. 2000). There are $N_{Z}=65$ redshift slices between $\bar{z}=0.2$ and 2.12 , and $N_{K}=25$ angular separation bins between 0.3 and $15^{\prime}$.

The galaxy redshift distribution follows the parameterisation suggested by Smail et al. (1995), i.e. $p(\bar{z})=$ $\beta /\left[z_{0} \Gamma_{\beta}(3 / \beta)\right]\left(\bar{z} / z_{0}\right)^{2} \exp \left(-\left(\bar{z} / z_{0}\right)^{\beta}\right)$, where $\Gamma_{\beta}$ denotes the gamma function. We take $\beta=3 / 2$ and $z_{0}=2 / 3$ yielding $\langle\bar{z}\rangle \approx 1$.

Two applications of the technique are presented. We start by asking how well the intrinsic and lensing contributions to the galaxy ellipticity correlation function can be separated, using 


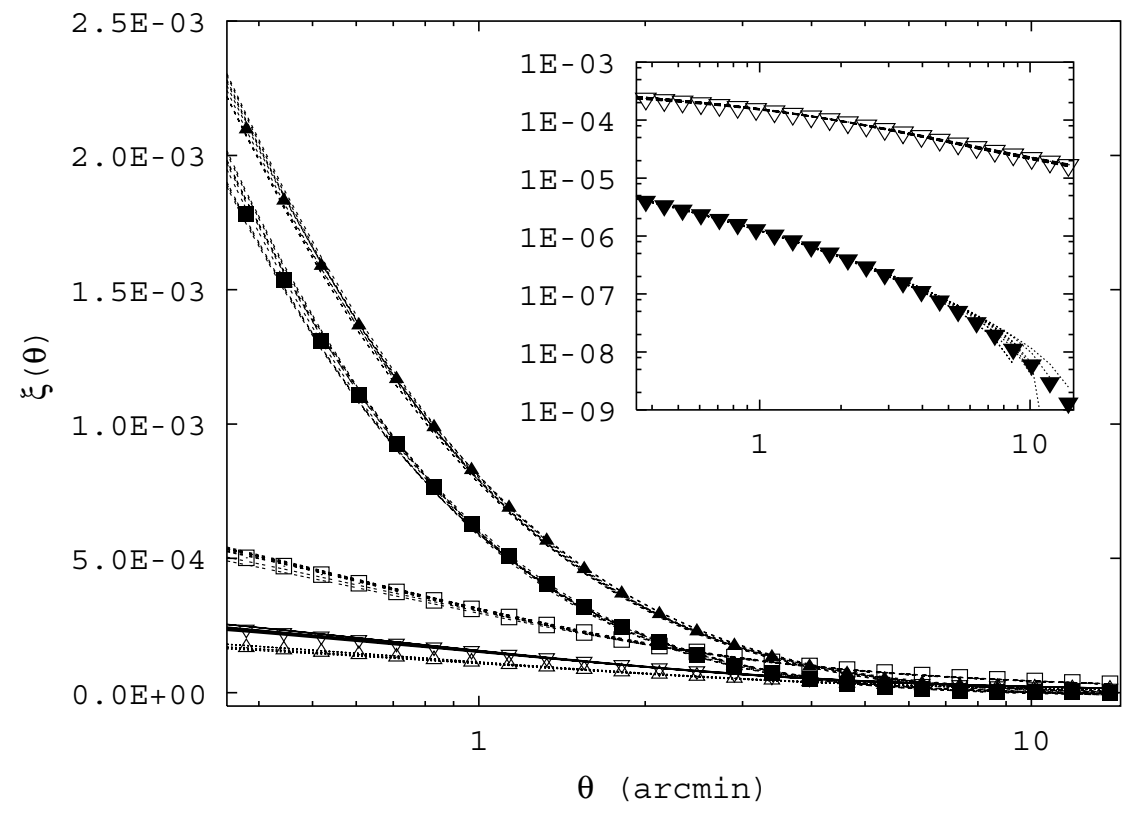

Fig. 2. The lensing and intrinsic cross-correlation functions measured between three pairs of photometric redshift bins for the $\Lambda \mathrm{CDM}$ cosmology and HRH intrinsic alignment model. Open (filled) squares correspond to the true lensing (intrinsic) signal for bins centred on $\bar{z}_{i}=1.07$ and $\bar{z}_{i}=1.13$. Open (filled) triangles show the true lensing (intrinsic) signal for bins centred on $\bar{z}_{i}=0.62$ and $\bar{z}_{i}=0.68$. Open (filled) inverted triangles show the true lensing (intrinsic) signal between bins centred on $\bar{z}_{i}=0.62$ and $\bar{z}_{i}=1.13$; since the intrinsic signal is so low, this is plotted on the inlay panel along with the lensing signal. The sets of lines associated with each of the true model symbols are the recovered best-fit lensing (intrinsic) cross-correlation functions to noisy realisations of $\bar{\xi}_{m}^{\text {obs }}$ for the pairs of photometric redshift bins, using the procedure described in the text.

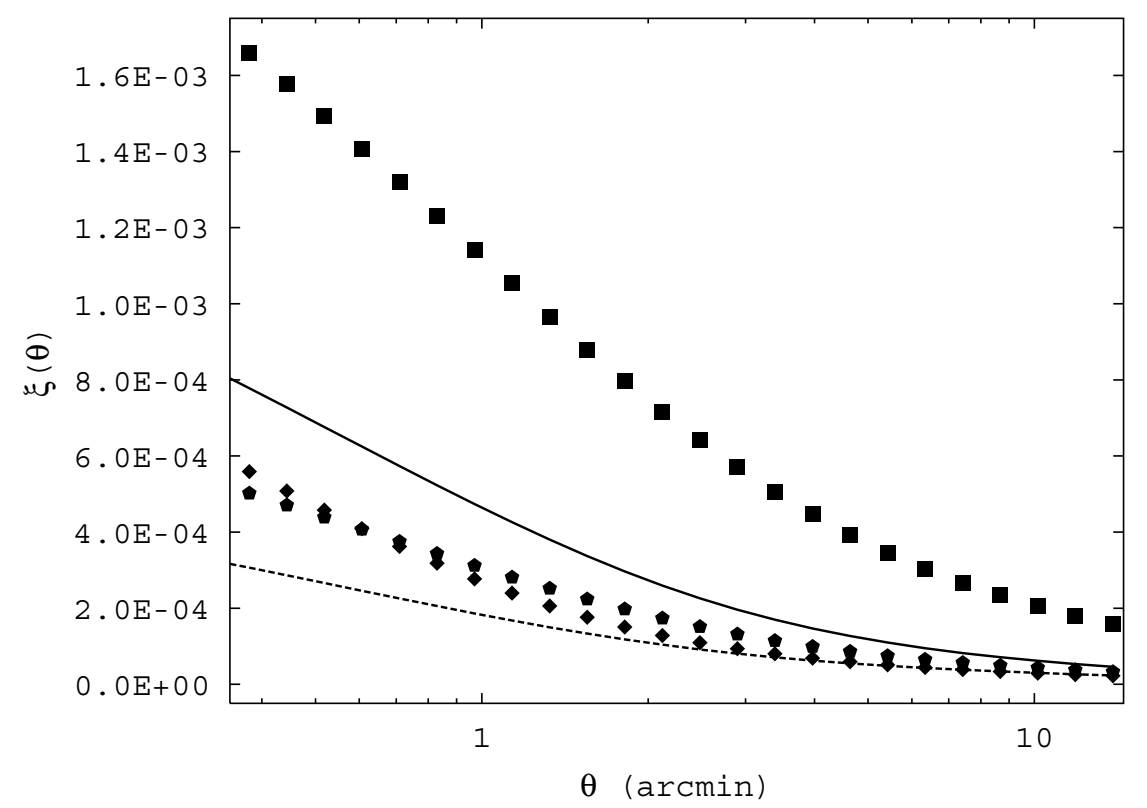

Fig. 3. The lensing correlation functions for the three cosmologies used for the construction of template functions, and for the other cosmologies considered in Sect. 3.1, plotted for the bins centred on $\bar{z}_{i}=1.07$ and $\bar{z}_{i}=1.13$. Filled squares correspond to $\sigma \mathrm{CDM}$, the filled pentagons indicate $\Lambda \mathrm{CDM}$ and filled diamonds correspond to OCDM. The dashed and solid lines indicate the flat cosmologies with (i) $\sigma_{8}=0.71, \Omega_{\mathrm{m}}=0.33$ and $\Gamma=0.215$, and (ii) $\sigma_{8}=0.8, \Omega_{\mathrm{m}}=0.5$ and $\Gamma=0.3$, respectively. the information contained in correlation functions between different redshift slices. Secondly, it has been shown that the degeneracy between $\Omega_{\mathrm{m}}$ and $\sigma_{8}$ can be partly lifted when redshift estimates for source galaxies are available (e.g. van Waerbeke et al. 2002a). We illustrate the use of correlation function tomography in this respect.

\subsection{Isolating the intrinsic correlation signal}

Now we consider input "observed" correlation functions $\bar{\xi}_{m}^{\text {obs }}$ comprising a lensing and an intrinsic contribution, to see how well the individual signals are recovered in terms of template functions using (9). First it was checked that in the absence of noise, $\hat{\mathcal{G}}_{n} \equiv \mathcal{G}_{n}$, when $\overline{\boldsymbol{\xi}}^{\text {obs }}$ is composed of a lensing and an intrinsic model contained in the set of templates.
The intrinsic alignment model for spirals from $\mathrm{HRH}$ $\eta(r, z)=0.012 \exp \left(-r / 1.5 h^{-1} \mathrm{Mpc}\right)$ was then used to obtain $\bar{\xi}^{\mathrm{I}}$, and $\bar{\xi}^{\mathrm{L}}$ was calculated for a $\Lambda \mathrm{CDM}$ cosmology. Random gaussian distributed errors with dispersion $\sigma=C_{m m}^{0.5}$ were added to these correlation functions giving noise realisations, and bestfit parameters $\mathcal{G}_{n}$ were recovered for each of these. Figure 2 shows the (noise-free) input and recovered intrinsic and lensing correlation functions between three combinations of redshift slices: one close pair at $\bar{z} \sim 1$, one close pair at $\bar{z} \sim 0.6$, and the correlations between slices at $\bar{z} \sim 1$ and $\bar{z} \sim 0.6$. The lensing correlation function for each of the three cosmologies used in the construction of template functions is shown in Fig. 3, plotted for the slices at $\bar{z} \sim 1.0$. The intrinsic correlation signal surpasses the lensing signal out to several arcminutes for both the low-redshift and the high-redshift bins. Considering bins with a large separation in redshift reduces the intrinsic signal 

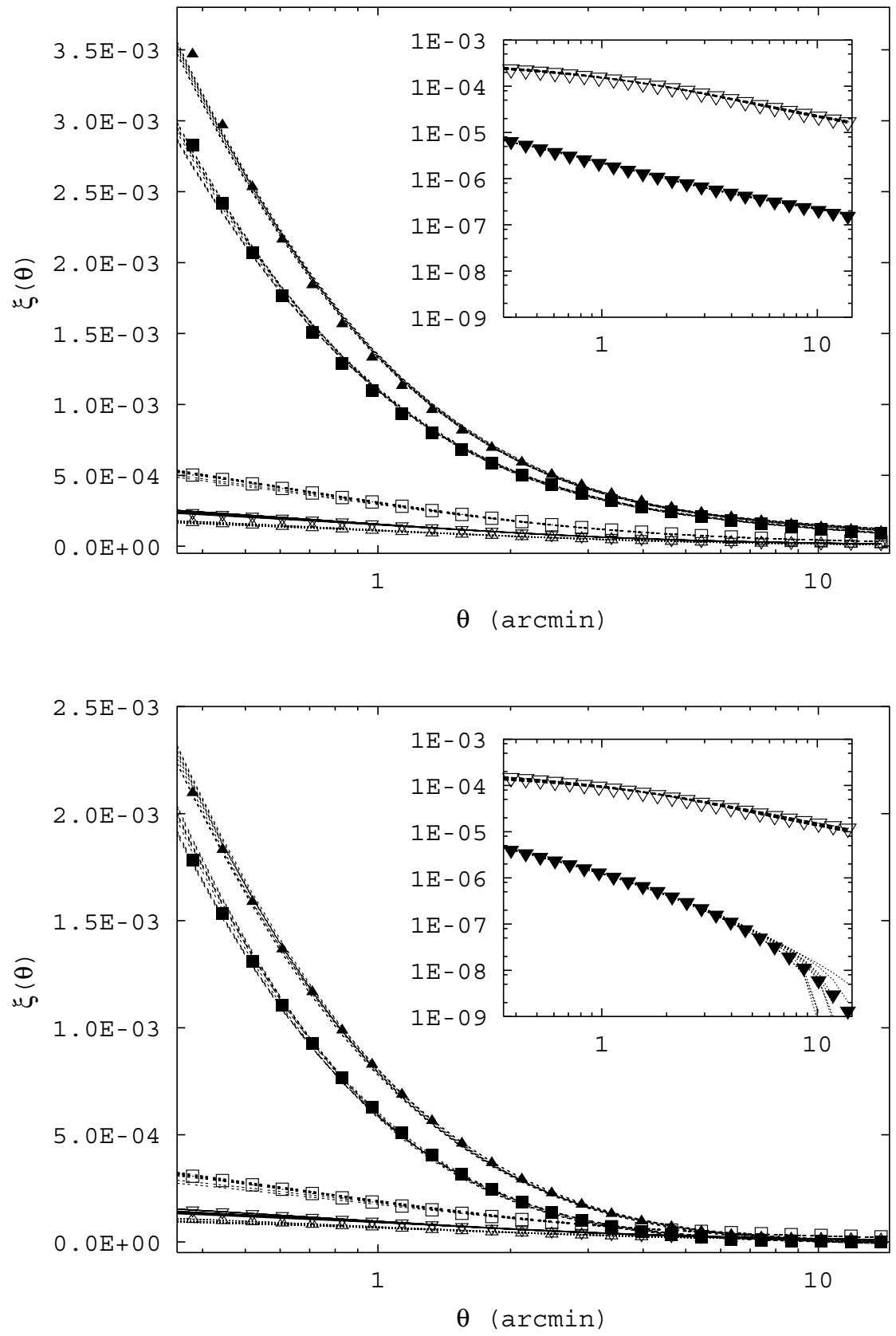

Fig. 4. The lensing and intrinsic correlation functions between different redshift bins for the $\Lambda \mathrm{CDM}$ cosmology and Jing (2002) intrinsic alignment model. The symbols and lines have the same meaning as in Fig. 2.

Fig. 5. The lensing and intrinsic correlation functions between different redshift bins for a flat cosmology with $\Omega_{\mathrm{m}}=0.33, \sigma_{8}=0.71$ and $\Gamma=0.215$ and an intrinsic alignment model from HRH. The symbols and lines have the same meaning as in Fig. 2.

to a negligible level, as expected. Even with our limited set of template functions, the reduced $\chi^{2}$ values of the recovered fits to the noise realisations are $\approx 1$. Also note that the intrinsic signal can be well represented in terms of the template functions, although it is not contained in the template set.

Since the current template set for the intrinsic alignment signal contains exponentially-decaying models with different scale-lengths, we now consider how well the method fares if the true signal is a power-law instead. The intrinsic alignment model is taken from Jing (2002); we use $\eta(r, z)=$ $0.288 /\left[r^{0.4}\left(7.5^{1.7}+r^{1.7}\right)\right]$, with $r$ measured in units of $h^{-1} \mathrm{Mpc}$. Again, the true cosmology is $\Lambda \mathrm{CDM}$. Noise was added and best-fit parameters recovered in the same manner as described above. Figure 4 shows the (noise-free) input and recovered intrinsic and lensing correlation functions between the same three combinations of redshift slices as for Fig. 2. Even though the functional form of the true intrinsic signal is quite different from the template models, the best-fit intrinsic models are still rather close to the noise-free model and more importantly, the lensing signal is again well recovered. To assess the difference between using the Jing model rather than the HRH model for intrinsic alignments, the reduced $\chi^{2}$ values of the recovered fits were determined for 1000 noise realisations of each, keeping the same $\Lambda C D M$ cosmology. The reduced $\chi^{2}$ value is lower for the HRH realisation in nearly all cases, since this is an exponentially-decaying model for which the templates are better adapted. The $\chi^{2}$ values for the HRH realisations closely follow the theoretically expected distribution. Although the intrinsic models can be distinguished statistically, the difference in the mean $\chi^{2}$ values of the two sets of 1000 realisations is only $\approx 15 \%$ of their dispersion. In practice, several families of functional forms could be taken for the template set. 


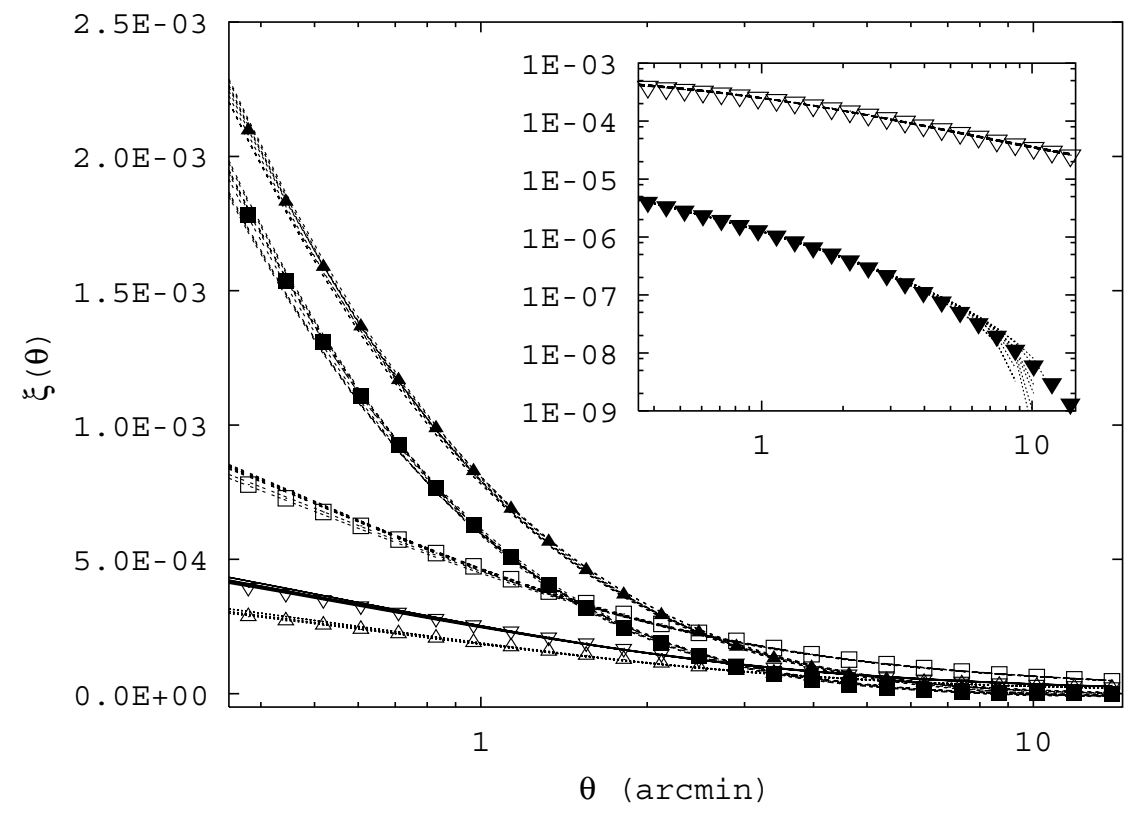

Fig. 6. The lensing and intrinsic correlation functions between different redshift bins for a flat cosmology with $\Omega_{\mathrm{m}}=0.5, \sigma_{8}=0.8$ and $\Gamma=0.3$ and an intrinsic alignment model from HRH. The symbols and lines have the same meaning as in Fig. 2.
The next example for $\bar{\xi}^{\text {obs }}$ again uses the HRH model for intrinsic alignments, but this time a different flat cosmology with $\sigma_{8}=0.71, \Omega_{\mathrm{m}}=0.33, \Gamma=0.215$ was used for the lensing correlations; the lensing signal for this cosmology was not part of the template set. Figure 3 shows the lensing correlation function for this cosmology, plotted for the slices at $\bar{z} \approx 1.0$. Noise was added using the same random seeds as above, and best-fit parameters recovered as before. Figure 5 shows results for the same combination of redshift slices as for Fig. 2. The power spectrum corresponding to this cosmology has a lower normalisation - the $\sim 20 \%$ reduction in $\sigma_{8}$ is not offset by the $10 \%$ increase in $\Omega_{\mathrm{m}}$; this is evident in the lower amplitude of the lensing correlation functions. Again, the recovered parameters are consistent with the true "observed" correlation functions and the reduced $\chi^{2}$ values $\approx 1$.

Whereas the foregoing cosmological model was quite different in amplitude of the power spectrum we now consider a model which also differs in the shape of the power spectrum, to test the robustness of our method. Keeping the HRH model for intrinsic alignments, a flat cosmology with $\sigma_{8}=0.8, \Omega_{\mathrm{m}}=0.5$, $\Gamma=0.3$ was next used for the lensing correlations. Figure 3 shows the lensing correlation function for this cosmology, plotted for the the slices at $\bar{z} \sim 1.0$. Noise was added and best-fit parameters recovered as described above. Figure 6 shows results for the same combination of redshift slices as for Fig. 2. The lensing signal is again well represented in terms of the basis functions, even though it is rather different to any of the cosmology templates. Hence, despite the fact that our set of template functions is quite restrictive, we have demonstrated that it provides enough flexibility to provide accurate fits to the correlation functions of quite different comological models.

\subsection{Breaking the $\Omega_{\mathrm{m}}-\sigma_{8}$ degeneracy}

The near degeneracy, in the absence of redshift information, between $\Omega_{\mathrm{m}}$ and $\sigma_{8}$ for two example flat cosmologies is illustrated in Fig. 7, taken from King \& Schneider (2002; where

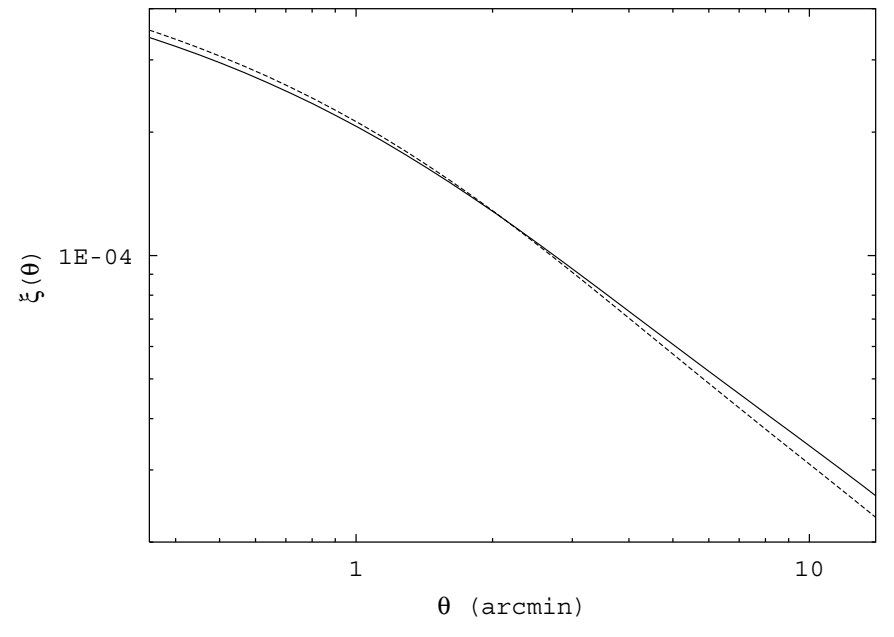

Fig. 7. This figure shows the lensing correlation functions for our fiducial $\Lambda$ CDM model: $\Omega_{\mathrm{m}}=0.3, \sigma_{8}=0.9$ (dashed line), and for a degenerate model with $\Omega_{\mathrm{m}}=0.4, \sigma_{8}=0.78$ (solid line). Both functions were predicted using the matter power spectrum described in Sect. 2.3, and for a redshift distribution with $\langle z\rangle=1$.

details for its calculation can be found). One cosmology is the fiducial $\Lambda$ CDM model: $\Omega_{\mathrm{m}}=0.3, \sigma_{8}=0.9$, and the other is an almost degenerate model with $\Omega_{\mathrm{m}}=0.4$ and $\sigma_{8}=0.78$. To obtain these correlation functions, we assume the same prescription for the power spectrum outlined in Sect. 2.3 above. The source population has a redshift probability distribution with $\langle z\rangle=1$, but with no individual photometric redshift estimates assumed to be available.

In order to see how well the fiducial and degenerate models could be distinguished using correlation function tomography, we took the gridded correlation functions for each model and added each of these to the gridded HRH intrinsic correlation function, giving $\left\langle\bar{\xi}^{\text {mod }}\right\rangle_{m}$ for each. For simplicity, we refer to these combinations as $\bar{\xi}_{\Lambda \mathrm{CDM}}$ and $\overline{\boldsymbol{\xi}}_{\text {degen }}$. A first set of simulations involved using a set of ten template functions 


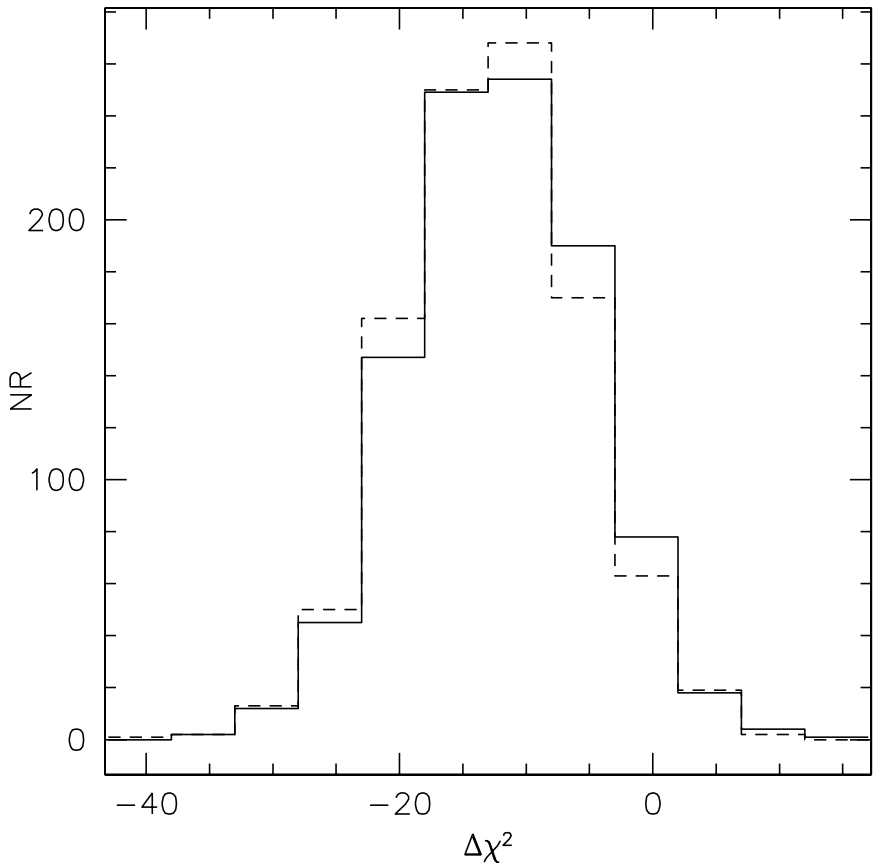

Fig. 8. These histograms show the frequency (NR) of values of $\Delta \chi^{2}$ for two sets of 1000 noise realisations. The first (second) set of realisations indicated by the solid (dashed) histogram has the fiducial (degenerate) $\Lambda \mathrm{CDM}$ cosmology in the template set and $\Delta \chi^{2}$ is calculated between the best-fit $\Lambda \mathrm{CDM}$ (degenerate) as opposed to degenerate $(\Lambda \mathrm{CDM})$ recovered model.

containing the nine models for intrinsic alignments, along with the $\Lambda \mathrm{CDM}$ lensing model for the lensing template. In turn, 1000 noise realisations of $\overline{\boldsymbol{\xi}}_{\Lambda \mathrm{CDM}}$ and $\overline{\boldsymbol{\xi}}_{\text {degen }}$ were generated using the same random seeds in both cases, and the bestfit amplitudes $\hat{\mathcal{G}}_{n}$ for the template functions recovered. The process was repeated, this time using the gridded degenerate model as the lensing template function, in place of the fiducial $\Lambda C D M$ model. The histograms of (i) $\Delta \chi^{2}(\Lambda C D M-$ degen $)$ and (ii) $\Delta \chi^{2}$ (degen $\left.-\Lambda C D M\right)$, corresponding to the difference in goodness-of-fit for the noise realisations when $\bar{\xi}_{\Lambda \mathrm{CDM}}$ and $\bar{\xi}_{\text {degen }}$ are used in the template set, are shown in Fig. 8. When the fiducial $\Lambda \mathrm{CDM}$ model (degenerate model) is the best-fit and is contained in the template set, values of $\Delta \chi^{2}$ in Fig. 8 should be negative. This gives a measure of our ability to differentiate between models using correlation functions between redshift slices. In the first histogram, when the model for $\overline{\boldsymbol{\xi}}_{\Lambda \mathrm{CDM}}$ is contained in the template set, in $95.6 \%$ of cases the noisy $\Lambda \mathrm{CDM}$ correlation functions are better fit. Also, when $\overline{\boldsymbol{\xi}}_{\text {degen }}$ is in the template set, $96 \%$ of the noisy degenerate correlation functions have better fits. Hence, within the assumptions we made and in the presence of an intrinsic alignment signal, these two cosmological models could be distinguished at the $\sim 2 \sigma$-level.

The foregoing example for distinguishing between two cosmological models should serve as a general illustration only. Owing to our simplified ansatz for the covariance matrix $\mathrm{C}$, which contains intrinsic ellipticity dispersion only, a more detailed investigation is not warranted here. In practice, cosmic variance would need to be taken into account in the covariance matrix when realistic constraints on cosmological parameters

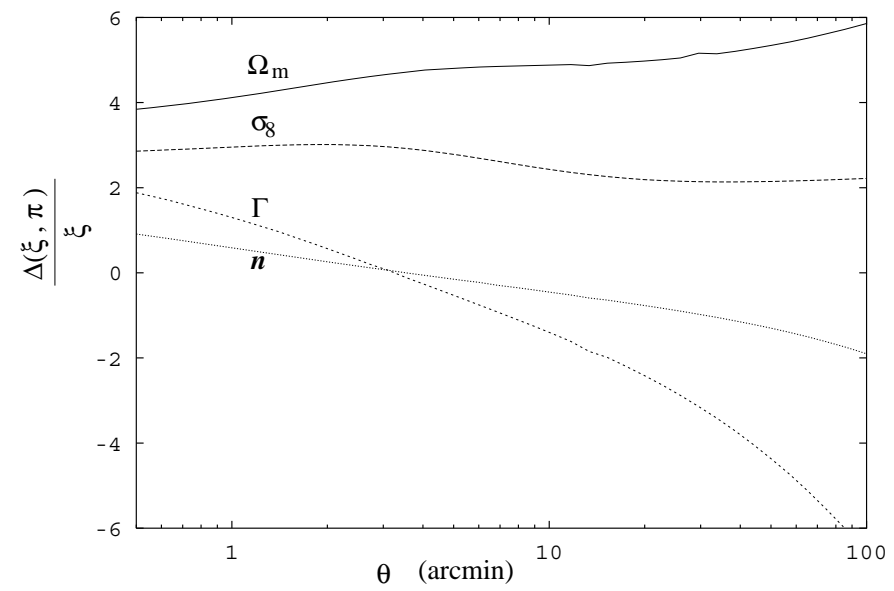

Fig. 9. The ratio of partial derivatives of $\xi$ to $\xi$ for our fiducial $\Lambda$ CDM model, with respect to parameters (i) $\Omega_{\mathrm{m}}$, (ii) $\sigma_{8}$, (iii) $\Gamma$ and (iv) $n$.

are to be derived. We are currently investigating ways to obtain a far more realistic representation of the covariance matrix, to be used in a study of the accuracy of cosmological parameter determination.

\section{Discussion and conclusions}

It has been suggested that the lensing correlation function may be contaminated by intrinsic galaxy alignments. Since cosmic shear probes the matter power spectrum and enables constraints to be placed on cosmological parameters such as $\sigma_{8}$ and $\Omega_{\mathrm{m}}$ (e.g. van Waerbeke et al. 2002a), it is vital to have the ability to isolate the contribution from intrinsic galaxy alignments in order to remove this systematic. Of course, intrinsic alignment is interesting in its own right: its amplitude as a function of physical separation and its evolution with redshift provides clues about the galaxy formation process.

We have demonstrated that measuring galaxy ellipticity correlation functions between redshift slices would enable the intrinsic and lensing contributions to be disentangled. The total signal is decomposed into template functions, and the fact that intrinsic alignments operate over a limited physical separation enables the intrinsic component to be isolated and subtracted from the total signal. Our knowledge of the amplitude of intrinsic alignments is limited, but no strong assumption about the behaviour of the intrinsic alignment signal needs to be made. Here we considered a modest number of template functions, which can easily be augmented to cover a wider range of functional forms. For example, any intrinsic alignment signal arising at the epoch of galaxy formation may be suppressed by subsequent dynamical interaction, perhaps most pertinent to galaxy pairs with extremely small physical separations. In fact, if the reduced $\chi^{2}$ of the best fit is significantly larger than 1 , this indicates that additional template functions need to be included.

Our choice of template functions is of course fairly arbitrary. We have taken functions which have approximately the behaviour expected from a cosmic shear measurement. Alternatively, one could consider a set of generic basis 
functions, which however, owing to the dependence on three variables, would require a fairly large set of functions. Another natural choice of the template functions could be the following: assuming a reasonable guess for the cosmological model, characterised by the parameters $\boldsymbol{\pi}_{0}$, the correlation function for neighbouring models could be written as

$\bar{\xi}\left(\theta, \bar{z}_{i}, \bar{z}_{j} ; \pi\right) \approx \bar{\xi}\left(\theta, \bar{z}_{i}, \bar{z}_{j} ; \pi_{0}\right)+\left(\boldsymbol{\pi}-\pi_{0}\right) \frac{\partial \bar{\xi}}{\partial \boldsymbol{\pi}}\left(\theta, \bar{z}_{i}, \bar{z}_{j}, \pi_{0}\right)$,

and therefore, the set consisting of $\bar{\xi}\left(\boldsymbol{\pi}_{0}\right)$ and its partial derivatives with respect to the relevant cosmological parameters would provide a useful set of template functions. As an illustration, in Fig. 9 we have plotted the partial derivatives of $\xi(\theta)$ for the case where no redshift information is available, i.e. the derivatives of the redshift-averaged correlation function, again with $\langle\bar{z}\rangle \sim 1$. For our cosmological model (characterised by $\boldsymbol{\pi}_{0}$ ) we take the fiducial $\Lambda$ CDM model. Derivatives are taken with respect to (i) $\Omega_{\mathrm{m}}$, (ii) $\sigma_{8}$, (iii) $\Gamma$ and (iv) the primordial spectral index $n$ (where our fiducial model is scale-invariant $n=1$ ). We plot the ratio $\frac{\partial \xi}{\partial \pi_{i}} / \xi$, where the numerator is denoted by $\Delta\left(\xi, \pi_{i}\right)$. In the limiting case where one curve is a scaled version of another, $\Delta\left(\xi, \pi_{i}\right) \propto \Delta\left(\xi, \pi_{j}\right)$, it is impossible to first order to break the degeneracy between parameters $\pi_{i}$ and $\pi_{j}$. We again see a nice illustration of the near degeneracy between $\Omega_{\mathrm{m}}$ and $\sigma_{8}$, manifest in the similarity between $\Delta\left(\xi, \Omega_{\mathrm{m}}\right)$ and $\Delta\left(\xi, \sigma_{8}\right)$. Since $\sigma_{8}^{2}$ enters into the linear power spectrum just as a prefactor, on large angular scales (i.e. in the linear regime), the curve for $\sigma_{8}$ tends to a constant, $\Delta\left(\xi, \sigma_{8}\right) / \xi \rightarrow 2 \sigma_{8}^{-1}$ for large separations. Another feature to note is that on the scale of a few arcminutes the curves for $\Gamma$ and $n$ change sign, implying that there is less degeneracy between these parameters and either of $\Omega_{\mathrm{m}}$ or $\sigma_{8}$.

There are several ways in which the method and results discussed here could be used. One way would be to consider the resulting split into intrinsic correlations and lensing signal as the final result, and to compare the resulting functions with theories of galaxies formation which predict the intrinsic alignment signal, and cosmological models predicting the shear correlation function. The resulting fits are, however, difficult to interpret statistically, i.e. the error bars on the shear correlation function are difficult to obtain. An alternative would be to consider the fitted intrinsic signal only, subtract it from the ellipticity correlation function, and consider the result as the shear correlation function, together with the corresponding error bars. Subsequently, the correlation function can then be used for the redshift-weighting method of King \& Schneider (2002), of course yielding much smaller contributions from the intrinsic correlations than for the unsubtracted data. Furthermore, the resulting model for the intrinsic correlation function could also be used as input for the subtraction method discussed in Heymans \& Heavens (2002).

In addition to providing a key to the suppression of any intrinsic alignment signal, photometric redshift estimates enable much tighter constraints to be placed on cosmological parameters obtained from cosmic shear surveys, as demonstrated by $\mathrm{Hu}$ (1999). Although our prime goal in this paper is not the constraint of cosmological parameters, we have illustrated that the degeneracy between $\Omega_{\mathrm{m}}$ and $\sigma_{8}$ can be lifted by observing correlation functions between redshift slices, even when an intrinsic alignment systematic is present.

Acknowledgements. We would like to thank Marco Lombardi, Patrick Simon, Douglas Clowe, and in particular Martin Kilbinger for helpful discussions. We would also like to thank the anonymous referee for very helpful remarks. This work was supported by the Deutsche Forschungsgemeinschaft under the project SCHN 342/3-1, and by the German Ministry for Science and Education (BMBF) through the DLR under the project 50 OR 0106.

\section{References}

Bacon, D. J., Refregier, A. R., \& Ellis, R. S. 2000, MNRAS, 318, 625 Bardeen, J. M., Bond, J. R., Kaiser, N., \& Szalay, A. S. 1986, ApJ, 304, 15

Blandford, R. D., Saust, A. B., Brainerd, T. G., \& Villumsen, J. V. 1991, MNRAS, 251, 600

Bolzonella, M., Miralles, J.-M., \& Pelló, R. 2000, A\&A, 363, 476

Brown, M. L., Taylor, A. N., Hambly, N. C., \& Dye, S. 2002, MNRAS, 333,501

Catelan, P., Kamionkowski, M., \& Blandford, R. D. 2001, MNRAS, 320L, 7

Crittenden, R. G., Natarajan, P., Pen, U.-L., \& Theuns, T. 2001, ApJ, 559,552

Croft, R., \& Metzler, C. 2000, ApJ, 545, 561

Hämmerle, H., Miralles, J.-M., Schneider, P., et al. 2002, A\&A, 385, 743

Heavens, A., Refregier, A., \& Heymans, C. 2000, MNRAS, 319, 649

Heymans, C., \& Heavens, A. 2002 [astro-ph/0208220]

Hoekstra, H., Yee, H., \& Gladders, M. 2002, ApJ, in press [astro-ph/0204295]

Hu, W. 1999, ApJ, 522, L21

Hui, L., \& Zhang, J. 2002 [astro-ph/0205512]

Jing, Y. P. 2002 [astro-ph/0206098]

Kaiser, N. 1992, ApJ, 388, 272

Kaiser, N. 1998, ApJ, 498, 26

Kaiser, N., Wilson, G., \& Luppino, G. 2000 [astro-ph/0003338]

King, L., \& Schneider, P. 2002, A\&A, 396, 411

Lahav, O., Bridle, S., Percival, W., et al. 2002, MNRAS, 333, 961

Mackey, J., White, M., \& Kamionkowski, M. 2002, MNRAS, 332, 788

Maoli, R., van Waerbeke, L., Mellier, Y., et al. 2001, A\&A, 368, 766

Mellier, Y., van Waerbeke, L., Bernardeau, F., \& Tereno, I. 2002 [astro-ph/0206434]

Miralda-Escudé, J. 1991, ApJ, 380, 1

Peacock, J. A., \& Dodds, S. J. 1996, MNRAS, 280, L19

Refregier, A., Rhodes, J., \& Groth, E. 2002, ApJ, 572, L131

Schneider P., van Waerbeke L., Jain B., \& Kruse G. 1998, MNRAS, 296, 873

Schneider, P., van Waerbeke, L., Kilbinger, M., \& Mellier, Y. 2002, A\&A, 396, 1

Smail, I., Hogg, D., Yan, L., \& Cohen, J. 1995, ApJ, 449L, 105

van Waerbeke, L., Mellier, Y., Erben, T., et al. 2000, A\&A, 358, 30

van Waerbeke, L., Mellier, Y., Pèllo, R., et al. 2002a

[astro-ph/0202503]

van Waerbeke, L., Mellier, Y., \& Tereno, I. 2002b

[astro-ph/0206245]

Wittman, D. M., Tyson, J. A., Kirkman, D., Dell'Antonio, I., \& Bernstein, G. 2000, Nature, 405, 143 